\title{
El ejercicio de la docencia virtual en la educación universitaria: consideraciones prácticas basadas en la experiencia de una asignatura para la formación de competencias informacionales
}

\author{
E-Learning in higher education: practical considerations drawn from a course on information skills development
}

\author{
Angels JiMÉnEZ LÓPEZ \\ Facultat de Ciències de la Comunicació, Universitat Autònoma de Barcelona, España \\ angels.jimenez@uab.cat
}

\begin{abstract}
Resumen
El artículo tiene como objetivo general ofrecer una serie de consideraciones prácticas orientadas a facilitar el ejercicio de la docencia virtual en instituciones de educación superior. Se parte de la experiencia adquirida en una asignatura destinada a la formación de competencias informacionales, para efectuar un análisis de los aspectos a considerar a la hora de afrontar este tipo de docencia. Aborda las características del espacio virtual, el perfil del profesor y del alumno, la comunicación e interacción en el aula y cuestiones relativas a la impartición de las materias. Expone problemas encontrados y soluciones dadas a los mismos, en la asignatura de referencia.
\end{abstract}

Palabras clave: Docencia virtual. Aprendizaje virtual. Buenas prácticas. Competencias informacionales. Educación superior. Universidades.

\section{Introducción}

La universidad tomó pronto conciencia del potencial de las tecnologías de la información y comunicación (TIC) en el campo educativo. Esto trajo consigo una reflexión encaminada a definir un nuevo modelo de institución superior en el que esas tecnologías fueran aprovechadas para mejorar la calidad de la docencia, el aprendizaje, la investigación, así como de los procesos de gestión administrativa derivados de tales actividades.

Entre las numerosas repercusiones de las TIC en el contexto universitario se encuentra el haber favorecido la colaboración entre instituciones. Así, por ejemplo, contribuyeron a la puesta en marcha de programas interuniversitarios de enseñanza virtual, cuya pretensión era diversificar y potenciar la multidisciplinariedad de la formación del alumnado. En dichos programas, cada centro ofrece una serie de asignaturas que

\begin{abstract}
Some practical considerations and advices to facilitate online learning in higher education institutions are drawn from the author's experience designing and developing a course on information skills development. Several key aspects are discussed: the characteristics of the virtual space, the teacher and students' profiles, communication and interaction among them, and the specific features of the subject affecting the learning process. The problems that were encountered and the solutions that were adopted are presented and analyzed.
\end{abstract}

Keywords: Virtual teaching. E-learning. Good practice. Information skills. Higher education.

los estudiantes de cualquiera de ellos pueden cursar, con independencia de su universidad de origen. Intercampus, Metacampus o Campus Global fueron algunos de los programas creados.

Aunque las TIC se encuentran plenamente instaladas en las instituciones de educación superior, centrándonos en la labor del profesorado, cabe señalar que su uso no siempre va acompañado de una innovación en consonancia con el potencial de las mismas. En algunos casos, esto se debe a la falta de formación específica. El Espacio Europeo de Educación Superior (EEES), así como la renovación de metodologías docentes y sistemas de aprendizaje promovidos por la Declaración de Bolonia, abocan cada vez más al uso de las TIC y las plataformas virtuales como sustento exclusivo de la docencia o como complemento a la misma. Por ello, iniciativas destinadas a la mejora de las 
competencias en este sentido, de profesores y alumnos, se hacen imprescindibles.

\section{Objetivos}

El presente trabajo parte de la voluntad de compartir la experiencia obtenida, tras varios años de docencia virtual, en una asignatura cuya principal finalidad es la formación de competencias informacionales. Esa experiencia, así como las características de la asignatura, han permitido establecer los siguientes objetivos:

- Señalar la necesidad de la formación en competencias informacionales en la educación universitaria. En una sociedad impelida por la tecnología, la información y el conocimiento, el aprendizaje a lo largo de la vida es uno de los pilares sobre los que se sustenta la competitividad profesional. Por ello, es imprescindible dotar a profesores y alumnos de los conocimientos y herramientas que les habiliten para llevarlo a cabo de manera óptima.

- Ofrecer algunas reflexiones, sobre la docencia virtual, partiendo de una experiencia docente. Desde el curso 2005-06 se imparte, en la Universitat Autònoma de Barcelona (UAB), la asignatura virtual de libre elección Surfear por el alud informativo de Internet 0 como buscar y organizar información en entornos digitales (en adelante "Surfear"). Tras siete años de ejercicio se ha obtenido una experiencia que se considera útil para otros docentes. Por ello, se ha creído oportuno darla a conocer tomándola como punto de referencia en las consideraciones presentadas en este artículo.

- Ofrecer una serie de orientaciones para la docencia virtual. Su finalidad es contribuir a las buenas prácticas. Se abordan aspectos sobre organización de aulas virtuales, comunicativos, elaboración de materiales didácticos, recursos utilizados, así como algunos de los principales problemas surgidos y las soluciones dadas a los mismos, en el marco de la asignatura de referencia - sobre buenas prácticas de e-learning, véase Cabrero y Romero (2010).

- Ayudar a los docentes en su aproximación a este tipo de docencia. El presente trabajo se propone también, entre sus objetivos, el facilitar el inicio del ejercicio de la docencia virtual al profesorado novel en esta modalidad.

\section{Significación de las competencias informacionales}

Las Normas sobre aptitudes para el acceso y uso de la información en la enseñanza superior (ACRL, 2000) las definen con claridad:

Conjunto de conocimientos, habilidades, disposiciones y conductas que capacitan a los individuos para reconocer cuándo necesitan información, dónde localizarla, cómo evaluar su idoneidad y darle el uso adecuado de acuerdo con el problema que se les plantea.

El mismo documento establece cuáles son esas aptitudes a través de cinco normas (Tabla I).

1. Determinar la naturaleza y nivel de la información que se necesita.

2. Acceder a la información requerida de manera eficaz y eficiente.

3. Evaluar la información y sus fuentes de forma crítica e incorporar la información seleccionada a su propia base de conocimientos y a su sistema de valores.

4. A título individual o como miembro de un grupo, utilizar la información eficazmente para cumplir un propósito específico.

5. Comprender los problemas y cuestiones económicas, legales y sociales que rodean al uso de la información, así como acceder y utilizar la información de forma ética y legal.

Tabla I. Competencias informacionales según la Association of College \& Research Libraries (ACRL, 2000)

Se trata de competencias fundamentales en el proceso formativo de cualquier individuo (ACRL, 2000):

Las aptitudes para el acceso y uso de la información constituyen la base para el aprendizaje continuo a lo largo de toda la vida. Son comunes a todas las disciplinas, a todos los entornos de aprendizaje y a todos los niveles de educación. Capacitan a quien aprende para dominar el contenido y ampliar sus investigaciones, para hacerse más autodirigido y asumir un mayor control sobre su propio proceso de aprendizaje"

\section{La docencia virtual}

Esta docencia presenta diversas especificidades que la diferencian de la presencial. En el presente artículo se tratarán las relacionadas con el espacio docente, el perfil del profesor, el perfil del alumno, la impartición de la docencia, y la comunicación e interacción en el aula. 
Más adelante se abordarán estos aspectos tomando como base la experiencia de la asignatura "Surfear". Como paso previo y para facilitar la contextualización, en el siguiente apartado se ofrece una somera descripción de la misma.

\subsection{Perfil de la asignatura}

El objetivo principal de "Surfear" es formar en la búsqueda avanzada de información a través de Internet, cuenta con 6 créditos y es de libre elección. Comenzó a impartirse en el curso 2005-2006 y se integra en los denominados "Programas de estudios compartidos en red". Estos son de carácter interuniversitario y fueron creados para potenciar el desarrollo de la universidad electrónica y el intercambio de asignaturas entre instituciones. Ofertan materias de libre elección que complementan y diversifican la formación del alumno. Estos programas son:

- Intercampus: Comenzó en el curso 1999-00. Lo comparten, aun hoy, las siete universidades públicas catalanas y la UOC. Ofrece 50 asignaturas. Cada institución imparte las que le corresponden desde su propia plataforma virtual. Los alumnos de cualquiera de las universidades pueden cursarlas con independencia de su centro de origen.

- Campus Global: Supone un paso más en el intercambio, al ampliar el alcance geográfico a universidades de diferentes comunidades autónomas (Politécnica de Madrid y UAB). Oferta 13 asignaturas.

- Metacampus: Se inició en el curso 19992000 y ofrece 8 materias compartidas entre la Universitat Oberta de Catalunya (UOC) y la UAB.

La Autónoma creó además Intracampus, programa dirigido exclusivamente a sus propios estudiantes.

"Surfear" se ha impartido en Intracampus (un grupo limitado a 40 alumnos) desde el curso 2005-06 al 2010-11 y en Intercampus desde 2006-07 a 2010-11 (grupo también limitado a 40 alumnos). Actualmente, curso 2011-12, se cuenta con un solo grupo de 40 estudiantes en Metacampus. Los recortes presupuestarios derivados de las medidas anticrisis han impuesto la reducción o extinción de algunos programas. Así por ejemplo, la UAB ha suprimido Intracampus.

Cabe señalar que, durante su andadura, la materia cubrió siempre la totalidad de plazas ofertadas e incluso las sobrepasó en demanda, hecho que refleja el interés que despertaron en el alumnado los contenidos propuestos.

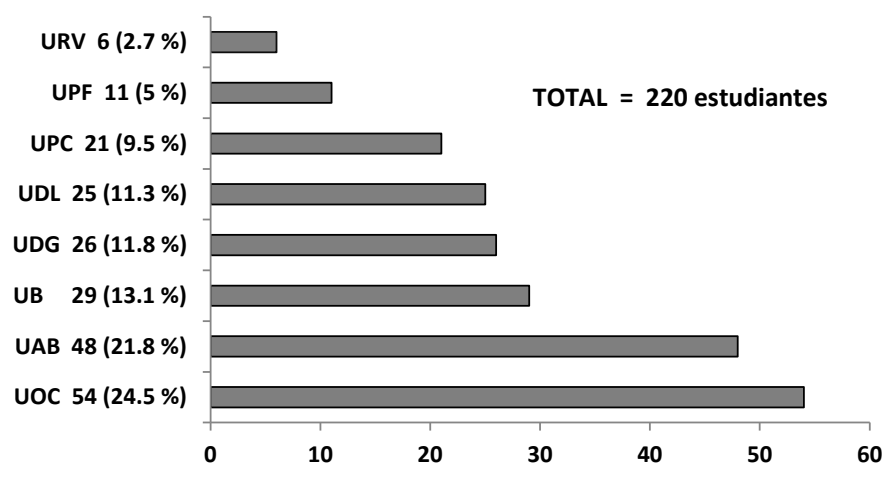

Figura 1. Número de alumnos matriculados en la asignatura entre 2006-07 y 2010-11 dentro de los programas interuniversitarios y porcentaje de participación por universidades (elaboración propia a partir de datos facilitados por la UAB)

\subsection{El espacio docente}

En la docencia virtual este espacio suele ser un aula sustentada por una plataforma tecnológica o campus virtual. En el caso de la UAB su responsable es la Oficina Autónoma Interactiva Docente, encargada del desarrollo y aplicación de las TIC en la institución, así como de dar soporte al usuario.

Para una enseñanza de calidad, la plataforma utilizada debería contar con prestaciones suficientes para cubrir el máximo número de tareas asociadas a la docencia y el aprendizaje. Al objeto del presente trabajo, y a modo de ejemplo, se destacarán exclusivamente aquellas utilidades de la plataforma de la UAB que facilitan la labor del profesorado:

- Agenda personal.

- Mensajería: Su existencia evita, por ejemplo, la recepción de mensajes del alumnado en el correo electrónico institucional del docente y permite disponer, en un único espacio, de todas las comunicaciones emitidas por los estudiantes.

- Sección de noticias: Funciona a modo de tablón de anuncios. Es útil para la notificación de cambios, la publicación de informaciones varias, avisos, etc.

- Banco de exámenes: Permite diseñar pruebas eligiendo los enunciados en un banco de preguntas previamente creado.

- Banco de preguntas: Es un repositorio que sirve de fuente para el diseño de exámenes. Puede incluir diversos tipos de cuestiones (verdadero/falso, opción múltiple, de texto 
abierto, etc.). Es posible incorporar preguntas mediante importación masiva.

- Herramienta de notas: Permite definir el sistema de evaluación (valor en porcentaje de las diferentes pruebas, puntuaciones de corte) y calcular los resultados finales. Puede trabajar con calificaciones importadas automáticamente desde otras aplicaciones en las que se generan notas como son la de entrega de trabajos y la de evaluación.

- Evaluación: Es un instrumento para la creación de convocatorias de examen. Pueden definirse los destinatarios, la fecha de realización y la duración, la fecha de publicación de los resultados, el tipo de corrección (automática o manual) y la exportación o no, de las calificaciones, a la herramienta de notas.

- Gestor de archivos: Es un espacio destinado al almacenaje organizado de los materiales que se utilizarán en la asignatura. Al finalizar un curso puede transferirse todo su contenido al espacio virtual del curso siguiente.

- Gestor de grupos: utilizada para realizar acciones y actividades diversas con equipos de trabajo previamente conformados por los alumnos (envío de mensajes colectivos, seguimiento de actividades, etc.).

- Entrega de trabajos: Es una herramienta para la recepción de ejercicios. Su funcionamiento consiste en la apertura de carpetas que actúan a modo de contenedores. Éstas pueden crearse de forma anticipada. La fecha en la que se harán visibles al alumno, así como el periodo en el que permanecerán abiertas, también pueden ser definidos de antemano, lo que ayuda a poder dejar las entregas establecidas incluso antes del inicio del curso.

- Foros: Son uno de los pilares de la interacción en el aula. Pueden servir con eficacia a distintos fines docentes, como por ejemplo, incorporar metodologías de aprendizaje entre iguales, fomentar la cohesión grupal, el conocimiento de los miembros del aula, favorecer la participación, etc. Constituyen una valiosa herramienta para obtener información acerca de la percepción que el alumnado está teniendo de la materia y de la docencia que se le imparte.

- Tutorías: Es una utilidad permite la realización de las mismas desde el espacio virtual. No es posible conversar en tiempo real, pero sí el intercambio de mensajes de uno a uno o de uno a varios, así como la adjunción de archivos.
La siguiente tabla resume las utilidades que desde el punto de vista docente, serían deseables en una plataforma virtual educativa.

Solidez tecnológica para la cobertura de todas o la mayoría de actividades asociadas a la docencia.

Máxima automatización de tareas para el ahorro de tiempo.

Posibilidad de planificación y programación anticipada de la docencia, es decir, dejar tareas realizadas incluso antes del inicio del curso (convocatorias de exámenes; apertura y cierre de foros, fechas de publicación de materiales, etc.) y que éstas se ejecuten automáticamente según el calendario establecido.

Diversidad de opciones para la comunicación e interacción en el aula.

Notificación automática de novedades cuando el docente entre al campus (aviso de recepción de mensajes, nuevas contribuciones publicadas en los foros, etc.).

Servicio eficiente de ayuda y soporte técnico que, además, informe puntualmente de las incidencias, como por ejemplo, horas o días de cierre del campus por motivos de mantenimiento $u$ otros.

Tabla II. Principales prestaciones exigibles a una plataforma virtual docente

\subsection{Perfil docente}

Afrontar la enseñanza virtual no es siempre fácil, en ocasiones se plantea como un reto. Los motivos pueden ser varios:

- La experiencia acumulada no es todavía muy amplia: Según lo que se desprende del estudio de 2001 La oferta de educación superior a través de Internet. Análisis de los campus virtuales de las universidades españolas, el arranque de las iniciativas de enseñanza virtual universitaria en España es relativamente reciente (Area, 2001, p. 153):

Hace una década prácticamente no existía ninguna oferta docente universitaria a través de Internet. Hace cinco años pocas universidades empezaron a experimentar una modalidad de oferta educativa mediante redes digitales. En el presente curso académico (2001-02) más de la mitad del conjunto de universidades españolas ofrecen formación superior a través de algún tipo de Campus Virtual.

Si se considera lo que representa instaurar y consolidar cambios de tal magnitud (elevadas inversiones en infraestructura tecnológica, aceptación y adaptación al uso de nuevas herramientas, creación de programas pa- 
ra la formación del profesorado, adecuación de los materiales de estudio, etc.) se entiende que dos décadas pueden ser todavía insuficientes.

- Las plataformas no siempre están preparadas para cubrir la totalidad o mayor parte de las tareas asociadas a la docencia. Con frecuencia, no se encuentran plenamente desarrolladas y van incorporando mejoras paulatinamente. Este hecho obliga a la utilización, algo incómoda, de herramientas cambiantes, así como a un seguimiento y adaptación frecuente, lo que se suma a las tareas habituales e incrementa la dedicación exigida por la labor docente.

- Falta de formación para la enseñanza virtual: Este tipo de docencia requiere competencias pedagógicas, informáticas, informacionales, metodológicas y comunicativas, no siempre coincidentes con las exigidas por la enseñanza presencial. La mayoría de universidades cuentan con programas de formación. Sin embargo, no son pocos los profesores que, por uno u otro motivo, se ven enfrentados a materias virtuales sin una preparación previa. Esto puede generar, por ejemplo, infrautilización de las herramientas, aulas virtuales en ocasiones algo desorganizadas, sensación de desbordamiento ante un incremento inesperado del volumen de trabajo (mensajería, dinamización de foros, adaptación de materiales...), sentimiento de incompetencia ante posibles quejas de los estudiantes, etc. Es conveniente no subestimar las implicaciones de este modo de enseñanza. En este sentido, la formación y la participación en actividades de intercambio de experiencias docentes son esenciales.

Los rasgos del profesor virtual han sido abordados ampliamente en la literatura especializada (v. g. Bautista et al., 2006; Casamayor, 2008; Silva Quiroz, 2010) y suelen ser bastante coincidentes en la mayoría de autores. Existen trabajos publicados que analizan su rol de facilitador, dinamizador y motivador del aprendizaje; asimismo, otros que describen en profundidad las competencias con las que debe contar. Por ello, y al objeto del presente trabajo, sólo serán enunciados de forma resumida, siguiendo la clasificación de Matabay (2008) (Tabla III).

Algunas de estas características son innatas. Sin embargo, en su mayoría pueden adquirirse con formación y práctica profesional.

Mantener una actitud reflexiva entorno a la docencia propia, ejercer la autocrítica y proveerse de canales para conocer la percepción y opinión del alumno acerca de la enseñanza que se le imparte, ayudará al profesor a obtener una radiografía bastante precisa de la calidad y efectividad de su trabajo.

Cognitivos

Experto en la materia que imparte.

Conocedor de los recursos y fuentes que permitirán al alumno adquirir las competencias previstas.

Poseedor de competencias informáticas e informacionales avanzadas.

Con buen dominio de las estrategias y métodos didácticos para entornos virtuales (técnicas de trabajo individual y colaborativo, sistemas de evaluación, etc.).

Conocedor de la forma en que aprenden los alumnos, del mecanismo del aprendizaje.

\section{Afectivos}

Comprometido éticamente con la labor educativa. Respetuoso con la autonomía del alumno en el proceso de aprendizaje.

Empático en la relación personal y pedagógica. Creativo, capaz de imaginar distintos escenarios de aprendizaje utilizando herramientas virtuales.

Positivo, motivador del interés del alumno hacia el aprendizaje.

Sistemático, con buena capacidad de organización y gestión del tiempo.

Proactivo, sensible en la percepción de problemáticas o dinámicas de grupo inadecuadas y eficaz en la resolución de las mismas.

Abierto a compartir sus experiencias con otros docentes para generar buenas prácticas profesionales.

Comunicativos o interactivos

Conocedor de las habilidades comunicativas propias de entornos virtuales y con buen dominio de la lengua escrita.

Perseverante en la retroalimentación del trabajo del alumno, puntual en sus respuestas y en el cumplimiento de sus compromisos docentes.

Tabla III. Rasgos del docente virtual (elaboración propia basada en Matabay, 2008)

\subsection{Perfil del alumnado}

Actualmente, las nuevas generaciones llegan a las aulas universitarias muy familiarizadas con el uso de las TIC. Son, en su mayoría, nativos digitales que han crecido con ellas al alcance. Tal vez ese sea el rasgo más característico del perfil del alumnado en general. Sin embargo, y salvo excepciones, eso no se traduce siempre en la posesión de competencias tecnológicas e informacionales óptimas para el ejercicio de su profesión futura y el aprendizaje a lo largo de la 
vida. Por ello, es necesario que adquieran esas competencias, además de las propias de la titulación que cursan.

La experiencia en la asignatura "Surfear" puso de manifiesto que la familiaridad con lo digital favorece, en gran medida, la rápida adaptación del alumno a los entornos virtuales, aunque es frecuente que matricule asignaturas de esta naturaleza sin conocer bien la plataforma tecnológica utilizada, sin haber cursado antes ninguna otra en esa modalidad, ni haber recibido formación específica. Esto plantea algunos inconvenientes y puede repercutir no sólo en su aprendizaje, sino también en la labor del docente. Un perfil óptimo del alumno virtual podría concretarse en las características indicadas en la tabla IV.

Competencias informacionales e informáticas básicas adquiridas.

Formado en el uso del campus virtual de su universidad.

Buena predisposición y voluntad para el aprendizaje autónomo (implicación y compromiso individual).

Buen dominio de la lengua escrita.

Colaborativo y participativo, consciente de que su aportación es importante para la construcción y adquisición conjunta de conocimiento (implicación y compromiso grupal).

Buen gestor de su tiempo.

Tabla IV. Perfil deseable del alumno virtual

En ocasiones, las características de algunas asignaturas determinan la coexistencia de perfiles bastante diferenciados en el seno de las mismas. Esto plantea al docente algunas cuestiones que deberá considerar tanto a la hora de planificarlas como de impartirlas. Una variedad de perfiles que no distorsione en exceso la homogeneidad necesaria para hacer viable la docencia y el aprendizaje será siempre enriquecedora. Un ejemplo de lo expuesto lo constituye "Surfear". Ésta presenta dos factores determinantes del perfil de su alumnado: su carácter de libre elección y su adscripción a programas intra e interuniversitarios. En Intracampus contaba sólo con estudiantes de la UAB pero de diferentes titulaciones y cursos. No eran pocos los que cursaban por primera vez una asignatura virtual y la mayoría conocía sólo medianamente las herramientas de la plataforma virtual. En Intercampus el alumnado procedía de ocho universidades distintas, de un amplio abanico de titulaciones y también de diferentes cursos. Desco- nocía el campus de la Autónoma. En Metacampus se cuenta con alumnos procedentes de la UOC (desconocedores del campus de la UAB pero habituados al trabajo en plataformas virtuales), así como con estudiantes de la Autónoma (con un conocimiento medio de la herramienta). En ambos casos son también de cursos y titulaciones diversas.

La figura 2 muestra el número de estudiantes que se matriculó la asignatura dentro de cada programa y durante qué tiempo se impartió en cada uno de ellos. Tratándose de una materia de libre elección, 462 alumnos totales en siete cursos es una cifra que refleja un alto grado de interés por la adquisición de competencias informacionales. Sobre todo, si se considera que, dada la situación de crisis que afecta también a las universidades, en el 2011-12 se ha eliminado uno de los dos grupos que se ofertaban, (lo que ha dejado a un buen número de estudiantes sin posibilidad de cursarla, aun habiéndola solicitado).

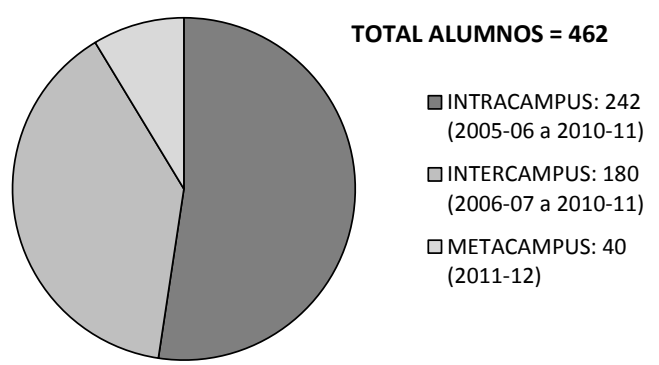

Figura 2. Número total de estudiantes matriculados en la asignatura y distribución por programas (elaboración propia a partir de datos facilitados por la UAB)

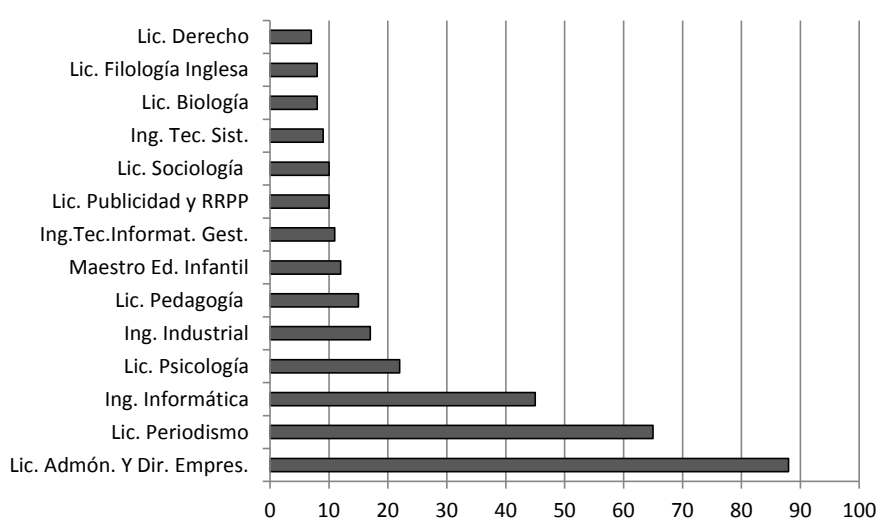

Figura 3. Procedencia del alumnado de la asignatura (elaboración propia a partir de datos facilitados por la UAB) 
La figura 3 muestra las titulaciones de las que proceden el mayor número de alumnos, lo que permite observar, además de la variedad de estudios de origen, en cuáles existe una mayor percepción de la necesidad de adquirir competencias informacionales. El ranking presenta las 14 especialidades con más de 5 alumnos matriculados. En total 327 estudiantes procedían de esas titulaciones y 135 quedaban dispersos entre otras tantas.

Los ámbitos empresarial, periodístico e informático son los que mayor matrícula generan. Esas son especialidades intensivas en la producción de información, pero también intensivas en su consumo. Sus estudiantes parecen ser conscientes de ello.

\subsection{Impartición de la docencia}

Este apartado no tiene la pretensión de explicar pormenorizadamente como se diseña, planifica o imparte una asignatura de modalidad virtual, sino ofrecer una serie de orientaciones prácticas basadas en la experiencia. Para ello, se señalan y comentan aquellas cuestiones que todo docente debería plantearse a la hora de afrontar este tipo de materias. Esas cuestiones son:

\subsection{1. ¿Se cursará la materia íntegramente a través del ordenador?}

Según lo constatado en "Surfear", la mayoría de estudiantes espera poder cursar estas asignaturas exclusivamente desde la virtualidad. Esta puede considerarse una actitud que limita en exceso el diseño de aquellas materias para las que no existen recursos didácticos abundantes, variados y de calidad en formato digital, pero lo cierto es que se da. Determinar este punto e informarlo en la guía de la asignatura evitará algunos problemas. El docente es libre, por ejemplo, de encargar la consulta de bibliografía disponible en la biblioteca "física" del centro o la realización de alguna actividad no virtual, pero debe saber que, si el estudiante no ha tenido forma de conocer eso antes de matricularse, probablemente se quejará. Cuando las quejas son manifestadas a través de comentarios en los foros, tienden a crear estados de opinión que repercuten negativamente en la dinámica constructiva del aula. Llegado a ese punto, el docente tendrá varias alternativas: convertir la actividad en voluntaria, mantenerla a pesar de las quejas o proponer un nuevo ejercicio íntegramente virtual. Sin embargo, lo mejor para evitar esas situaciones es anticiparse, plantearse y tomar de antemano la decisión que se considere más oportuna acerca de la forma en que se podrá cursar la materia.
En el caso de "Surfear", tras haber experimentado la problemática descrita durante el primer año en el que se impartió, se optó por la virtualidad total de las actividades obligatorias. No sin antes evaluar si se disponía de material didáctico suficiente, accesible, gratuito y de calidad. En algunos casos, fue necesaria la digitalización de artículos y otros documentos de lectura, pero dadas las características de la asignatura, no supuso mayores complicaciones.

\subsection{2. ¿Qué actividades se propondrán para evidenciar el aprendizaje y alcanzar los objetivos establecidos? ¿En qué medida son accesibles al alumno las herramientas tecnológicas necesarias para realizarlas?}

Esta cuestión guarda relación con la anterior pero el asunto que plantea es algo distinto y se expondrá a través de un ejemplo:

Uno de los objetivos de "Surfear" es que el alumno aprenda y practique la búsqueda documental en bases de datos especializadas. Las bibliotecas universitarias cuentan hoy en día con subscripción a un amplio número de ellas. Cuando la asignatura se incluyó en el programa Intercampus, el equipo docente sabía que contaría con alumnos de ocho universidades distintas. El hecho de que pudieran matricularse y tener acceso al campus de la Autóma hizo presuponer que los estudiantes de otras instituciones también tendrían privilegios de acceso a los recursos bibliotecarios digitales de la UAB. En base a ello se diseñaron actividades en las que se proponía la búsqueda de información en determinadas bases de datos. Craso error, porque llegado el momento de realizarlas, los alumnos externos carecían de acceso a las fuentes necesarias por problemas tecnológicos (según informó el servicio informático).

Este contratiempo imprevisto se resolvió elaborando una nueva actividad a partir de recursos accesibles a todos, pero sus consecuencias fueron, un trabajo añadido a los docentes y la consiguiente modificación del calendario de entrega de ejercicios.

La experiencia pone de manifiesto la necesidad de constatar la disponibilidad de las herramientas, fuentes y recursos que se vayan a utilizar a lo largo del curso, así como la revisión periódica de la misma.

\subsection{3. ¿Qué sistema de corrección de actividades se aplicará?}

Otro punto a decidir será la forma de corregir las actividades y ejercicios propuestos. En este sentido, es preciso considerar el número de 
alumnos y de actividades que se desea programar.

$\mathrm{Si}$ el grupo es muy numeroso y el profesorado escaso, pretender una corrección que incluya comentarios a cada uno de los trabajos presentados por el alumno será bastante difícil de asumir. El docente se verá obligado a pasar un excesivo y perjudicial número de horas ante la pantalla del ordenador. Una buena alternativa es elaborar solucionarios exhaustivos. Eso le permitirá un ahorro de tiempo, ya que sólo deberá revisar y calificar los ejercicios sin la necesidad de devolverlos con anotaciones. Además, obligará al alumno a comparar sus respuestas con las soluciones dadas, lo que resulta siempre una actividad muy formativa. Si tras esa comparación se le presentan dudas podrá exponerlas en un foro habilitado para tal fin y le serán resueltas por el profesor. De este modo se beneficiará también el resto del grupo.

Sin duda, el tiempo invertido en la confección de solucionarios detallados será ampliamente amortizado.

\subsection{4. ¿Cómo se diseñarán los materiales de estudio?}

La elaboración de materiales didácticos es, con toda probabilidad, lo que mayor esfuerzo exigirá al docente, sobre todo si imparte la asignatura por primera vez. Por lo general, el alumnado virtual no espera que los materiales se limiten a una colección de apuntes, meramente textuales, al modo tradicional. En su mayoría ha crecido en una sociedad multimedia y ese es uno de los lenguajes que mejor entiende. El profesor debe asumir esta realidad y adaptar sus herramientas, metodologías y recursos didácticos, para conseguir que sus enseñanzas sean adecuadamente transformadas en conocimiento adquirido.

Elaborar materiales no limitados al texto escrito es lo deseable. El principal inconveniente en este sentido, se encuentra en la obtención de documentos multimedia de calidad y aptos para la docencia. Dependiendo de los contenidos a impartir esto puede convertirse en algo complicado. En esos casos, puede optarse por la creación de los mismos. Sin embargo, esta es una alternativa que requiere tener los conocimientos necesarios, contar con colaboradores expertos dispuestos a prestar ayuda o, por lo general, disponer de recursos económicos para contratar personal experto que los desarrolle. Algunas universidades han creado programas de subvención a la innovación y mejora de la calidad docente, también existen convocatorias de instituciones públicas de la misma naturale- za. Así, por ejemplo, la Agència de Gestió d'Ajuts Universitaris i de Recerca (AGAUR) de la Generalitat de Catalunya, dispone del programa Millora de la Qualitat Docent (MQD) desde el que financia proyectos presentados por las universidades catalanas.

Cuando se planteó el diseño del material didáctico de "Surfear" se tomaron tres decisiones:

a) Para cada tema se elaboraría un único documento que incluiría todo lo necesario para que el estudiante pudiera trabajarlo (apuntes teóricos, ejercicios prácticos, lecturas, documentación complementaria, etc.).

b) Ese documento tendría, en lo máximo posible, un carácter multimedia. Incluiría o enlazaría videos, registros sonoros, presentaciones, tutoriales, gráficos interactivos, etc.

c) Se desarrollaría mediante el programa Power Point. Posiblemente un formato de página web hubiese sido más adecuado, pero en aquel momento las características de la plataforma virtual hacían compleja la publicación de páginas web dentro del campus.

El resultado final fue bastante satisfactorio, no sólo a juicio del equipo docente, sino también del alumnado, que lo elogió en sus valoraciones.

Por otro lado y aunque el estudiante disponía del programa de la asignatura, se consideró oportuno elaborar, además de los materiales estrictamente de estudio, diversos documentos que explicaran en detalle las características de la misma. Estos documentos fueron:

- Funcionamiento de la asignatura y del aula: Se trata de una presentación que aclara, entre otros aspectos, los relacionados con la dinámica de trabajo por periodos temporales y módulos temáticos, hace saber que se publicarán solucionarios pormenorizados de los ejercicios prácticos, informa sobre la forma de corrección y notificación de calificaciones, especifica la finalidad y uso de los diferentes canales de comunicación admitidos, así como cuáles son las normas básicas de convivencia en el aula.

- Normativa de presentación de trabajos: Incluye los formatos de archivo permitidos, el nombre uniforme que debe darse a los mismos para su rápida identificación, características de los documentos que se entreguen, formas aceptadas y no aceptadas de entrega, consecuencias de la presentación no acorde a las normas y plazos establecidos, etc. 
- Formulario de informe valorativo: Después de trabajar cada tema, se estableció que el alumno debería reflexionar y hacer una valoración de lo aprendido en el mismo. Para ello, respondería a un cuestionario (al que se denominó informe valorativo) planteado por el docente. Con el fin de que todos los informes fuesen homogéneos en su forma, se creó un documento que el estudiante utiliza a modo de plantilla en la realización de sus informes.

\subsection{5. ¿De qué forma se estructurará el aula?}

Dependiendo de la versatilidad y prestaciones de la plataforma virtual utilizada, el docente deberá encargarse, en mayor o menor medida, de conferir claridad y estructuración al aula, especialmente en lo que se refiere a organización y clasificación de los materiales didácticos e informativos publicados (guías, apuntes, enunciados de pràcticas, solucionarios, textos de lectura, referencias bibliográficas, enlaces a páginas web, videos, etc.). Esta es una tarea no exigida por los entornos presenciales y que tal vez resulte obvia, pero lo es sólo realtivamente. Cobra importancia cuando se cuenta con estudiantes que no conocen bien el funcionamiento del campus o provienen de otras universidades y por lo tanto, no lo han utilizado nunca. En esos casos dotar al espacio virtual de una organización clara evita la confusión. De hecho, es uno de los aspectos que el alumnado más suele comentar y evaluar positiva o negativamente, si se le solicita su opinión sobre la asignatura.

Algunas plataformas se presentan ya muy estructuradas, otras no tanto. En el caso del campus UAB existe una sección genérica, denominada Material Docente, que el profesor puede organizar a su conveniencia mediante un sistema de carpetas y subcarpetas. Se puede optar por una clasificación temática, abriendo un contenedor para cada tema en el que se incluirá todo lo referido al mismo con independencia de su tipología documental. Otra alternativa es la clasificación tipológica, según la naturaleza de los materiales (por ejemplo, presentaciones Power Point, referencias bibliográficas, artículos o documentos de lectura, enlaces web, etc.). Sin embargo, esta obliga a crear subdivisiones temáticas, para evitar la dispersión.

El sistema de clasificación idóneo atenderá al número y tipología de los materiales que se tenga previsto utilizar a lo largo de todo el curso. No es aconsejable crear numerosas carpetas y que un buen número de ellas acaben vacías o con sólo uno o dos documentos en su interior. Cuanto más simple sea la estructura clasificatoria, más efectiva será. Diseñarla antes de iniciar la docencia y ponerla a la vista del alumno desde el primer momento, le permitirá familiarizarse con la misma y localizar cualquier material de forma rápida, lo que redundará en una mejor comprensión y manejo del aula.

\subsection{6. ¿Cuáles serán las estrategias para conseguir una comunidad de aprendizaje operativa?}

El espacio virtual es, en principio, frío, impersonal y carente de la emotividad que caracteriza a la comunicación coincidente en espacio y tiempo, así como a la no verbal. Esto provoca un distanciamiento que conviene paliar en beneficio de la cohesión y operatividad del grupo. No obstante, es preciso señalar que la virtualidad potencia algo que muchos docentes extrañan en las aulas presenciales, la participación del alumno. El anonimato que proporciona relaja, distiende y desinhibe al estudiante, favoreciendo el planteamiento de dudas, la libre expresión de opiniones y la discusión de puntos de vista diferentes a los suyos.

Crear una comunidad de aprendizaje donde los individuos asuman que el nivel de adquisición de conocimientos depende, en buena medida, de todos, debe ser un ojetivo prioritario para el docente. Para su consecución se aconseja reflexionar las acciones que se llevarán a cabo o las iniciativas que se emprenderán en este sentido.

Es necesario posibilitar que los miembros del grupo se conozcan. Por ejemplo, en "Surfear", cuando comienza el curso y el alumno entra por primera vez al Campus, encuentra ya publicado un mensaje de bienvenida del equipo docente y un foro abierto, denominado "Quién es quién", para efectuar presentaciones (incluídas las del profesorado) - véase Cepillo (2010) para utilidades y usos de los foros. Como mínimo deben anotar nombre, universidad y titulación de procedencia, pero se les anima a incluir, de manera voluntaria, aficiones, preferencias, motivos que le llevaron a matricular la asignatura, espectativas de aprendizaje, nivel de competencias informacionales que creen poseer, etc. Las presentaciones deben hacerse en un plazo no muy extenso para conseguir que se conozcan a la mayor brevedad (una o dos semanas es suficiente cuando el grupo es muy numeroso). Los alumnos suelen ser proclives a proporcionar abundantes datos, lo que le da a este foro un carácter distendido y amigable que rompe buena parte del distanciamiento inicial.

Este espacio no sólo contribuye al conocimiento del grupo, también se convierte en una fuente de información muy útil para el docente acerca 
de las espectativas de sus alumnos. Eso puede ayudarle a orientar los contenidos o incidir en los aspectos que despiertan mayor interés, contribuyendo así, a hacer más atractiva la asignatura.

Igualmente, desde el inicio del curso, encuentran otro foro disponible pero en este caso de naturaleza lúdica. En él se anima a comentar, de forma voluntaria, informaciones de actualidad, sugerir películas, libros, música, a anunciar actividades culturales, etc. Resulta curioso y enriquecedor el dinamismo que adquiere este foro en algunos grupos. De él han surgido, incluso, convocatorias para ir al cine, salidas para hacer escalada o interesantes debates sobre algún hecho o acontecimiento del día.

También es posible potenciar la conciencia de comunidad y la cohesión grupal a través de intervenciones o mensajes que recuerden el valor de la participación y la colaboración. Por ejemplo, destacar acciones positivas resulta muy efectivo. Si en un foro un estudiante resuelve alguna duda planteada por otro, un oportuno comentario del docente ("Perfecta tu respuesta. Gracias por la ayuda") servirá, por un lado, para dejar constancia de su presencia en el aula y de su nivel de atención pero, sobre todo, para propiciar un buen clima de aprendizaje. Por lo general, el alumnado es sensible a este tipo de intervenciones y suele reaccionar a las mismas de forma muy positiva. El docente observará que la ayuda entre compañeros se hace más frecuente, lo que contribuye a un aprendizaje entre iguales que aporta siempre abundantes beneficios.

\subsection{7. ¿Qué tiempo de atención se dedicará al alumno?}

Cada profesor destinará a la atención de los estudiantes el tiempo que considere oportuno. En realidad, lo que se plantea en este punto es la necesidad de establecerlo e informarlo desde el inicio del curso.

Algunos alumnos, mayormente los que cursan por primera vez asignaturas virtuales, llegan con la idea, o tal vez la sensación, de que el docente está o debe estar "al otro lado" de forma casi permanente y esperan que cualquier duda que le remitan sea aclarada en el momento en el que ésta se suscita. Cuando no ocurre así, algunos manifiestan sus quejas a través de los foros. Para evitarlas, es suficiente con informar, desde el principio, cuáles son las normas en este sentido. Por ejemplo, el profesor puede establecer que responderá las cuestiones planteadas en el foro de dudas los lunes y jueves. Probablemente y como entrará bastantes más veces al campus a lo largo de la semana, también responderá otros días, pero el alumno debe saber que eso no es una obligación. En este sentido, lo aconsejable es la información anticipada.

\subsection{8. ¿Qué volumen y tiempo de trabajo le supondrá al alumno cursar la materia?}

Como en la docencia presencial, conviene hacer un cálculo aproximado de lo que representará, para un estudiante medio, el seguimiento de la asignatura. Al tiempo de estudio, realización de ejercicios, lecturas, etc., deberá sumársele otro, en consideración de las dificultades de tipo técnico que puedan surgir, las derivadas de la inexperiencia en el uso de las herramientas tecnológicas, etc. Conviene evidenciar al alumno que esas eventualidades se han tenido en cuenta en la planificación de la asignatura.

Por ejemplo, en el caso de "Surfear" y por la diversidad de procedencias, la primera semana se destina al conocimiento y adaptación del alumnado. Cuando este entra por primera vez al aula encuentra una noticia publicada en la que se informa que esa primera semana constituye el margen de tiempo previsto por el profesor, para conocer la dinámica y normas de la asignatura. En ese tiempo debe leer la documentación informativa al respecto y familiarizarse con la plataforma virtual si la desconoce (revisar tutoriales, practicar su uso, etc.). También debe consultarle al docente cualquier aspecto que no le haya quedado claro y efectuar su presentación, ante el resto de compañeros, en el foro "Quién es quién". Salvo excepciones, la mayoría realiza las tareas encomendadas puntualmente. Esto se observó por el mejor funcionamiento del aula en comparación con años precedentes en los que no se dedicó esa primera semana al propósito expuesto. Los beneficios que se consiguieron desde la perspectiva docente fueron varios:

- Homogeneizar el grupo antes de comenzar a trabajar los temas de la asignatura.

- Disminuir los errores, en general involuntarios, cometidos por el alumnado (por ejemplo, entrega de trabajos o envío de mesajes por canales inadecuados, etc.). Esto se tradujo para el profesorado en un ahorro de tiempo ya que el número de correos recibidos y emitidos, el de trabajos que debía identificar y reubicar por haber sido enviados de forma inadecuada, etc., fue mucho menor.

- Posibilidad de penalizar el incumplimiento de las normas, que tanto puede afectar al correcto funcionamiento de la asignatura: Des- 
pués de haber contado con un plazo exclusivo para conocer la dinámica de la misma, le quedan al estudiante muy pocas excusas para justificar actitudes, acciones o procedimientos al margen de la normativa establecida.

En definitiva y como se señaló al inicio de este apartado conviene hacer un cálculo aproximado del volumen y tiempo de trabajo que le supondra al alumno la asignatura, así como evidenciarle a este que el tiempo que puedan requerirle las especificidades propias del entorno virtual ha sido contemplado en dicho cálculo.

\subsection{9. ¿A través de qué canales se obtendrá información sobre la percepción de la asignatura que están teniendo los estudiantes?}

Considerar de qué modo se obtendrá información sobre las dificultades que el alumno va encontrando a lo largo del curso es necesario para resolverlas a tiempo.

La apertura de un foro específico de dudas sobre los contenidos de la materia constituye una muy buena opción pero puede no ser suficiente, ya que no todos los estudiantes se animan a utilizarlo. En el caso de "Surfear" ese foro existe. Es el denominado "Pasa palabra". Nada que no tenga que ver con los temas que se trabajan, cuestiones de calendario, entrega de ejercicios, etc., es decir, nada que no afecte a todo el grupo o de lo que pueda beneficiarse en su conjunto, podrá exponerse en el mismo. Los asuntos personales o que afectan a una única persona se tratan a través de la mensajería o de las tutorías del campus. En este foro, las dudas pueden ser resueltas por cualquier miembro del aula (el docente o los propios compañeros). Se determinó así para potenciar el aprendizaje entre iguales. No obstante, cabe señalar que, aunque tiene ventajas, exige al docente un seguimiento muy frecuente no sólo para aportar respuestas, sino también para detectar y rectificar posibles aclaraciones erróneas hechas por el alumnado.

En la asignatura se estableció, además, otro canal para tomarle el pulso al proceso de aprendizaje del grupo. Se trata de los "Informes valorativos" de cada tema. Como ya se apuntó, constituyen una herramienta de detección muy eficaz en este sentido.

Al finalizar el periodo establecido para trabajar un tema el alumno debe responder y entregar obligatoriamente al docente un cuestionario con preguntas relativas a seis cuestiones: conocimientos que cree haber adquirido, habilidades desarrolladas, tiempo de trabajo dedicado, aspectos de mayor dificultad, opinión sobre los materiales de estudio proporcionados y sugerencias que desee hacer.

Las utilidades de estos informes son varias:

1. Favorecen la reflexión del alumno sobre su propio proceso de aprendizaje.

2. Le ayudan a seguir el hilo conductor de la asignatura.

3. Aportan una información valiosa que el docente podrá utilizar para incidir en puntos que no hayan quedado claros a nivel grupal, incorporar mejoras en los materiales, etc., es decir, para resolver problemáticas o reconducir situaciones.

\subsubsection{0. ¿Qué instrumentos se utilizarán para evaluar el aprendizaje?}

Independientemente del sistema de evaluación por el que se opte, en la docencia totalmente virtual, la elección de los instrumentos para llevarla a cabo se ve condicionada por las herramientas disponibles para tal fin. La plataforma tecnológica debería permitir realizar exámenes, corregir automáticamente algunos tipos de pruebas, calcular calificaciones finales, etc. En su defecto, las posibilidades de evaluación se verán limitadas y ese es un factor a tener en cuenta a la hora de planificar la docencia.

A modo de ejemplo, en "Surfear" se optó por un sistema de evaluación continua en el que se valoran diversos aspectos. A cada uno de ellos se le asigna un porcentaje dentro de la calificación final:

- Seguimiento de la asignatura (20\%): Considera la puntualidad en la entrega de ejercicios prácticos y la calidad de los mismos, la realización de los informes valorativos y de actividades opcionales complementarias planteadas.

- Participación (10\%): Valora las contribuciones en los foros y debates propuestos.

- Nivel de competencias informacionales adquiridas (70\%): Hacia el final de la asignatura el alumno debe realizar un trabajo individual de síntesis en el que habrá de aplicar los conocimientos, destrezas y habilidades adquiridas a la resolución de un caso práctico (de temática diferente para cada estudiante). Su finalidad es evidenciar el nivel de competencias informacionales alcanzado. La materia no se supera si en este trabajo no se obtiene una calificación mínima de aprobado.

Se desestimó la realización de exámenes teóricos por dos razones, la primera, que por la naturaleza eminentemente práctica de la asignatura, 
lo que se deseaba priorizar en la evaluación era la capacidad adquirida para la resolución de casos y necesidades informativas dadas. La segunda, que la resolución óptima y bien argumentada de un supuesto como el que se les propone resulta casi inviable si no se han asimilado adecuadamente los contenidos de la materia. En este sentido el trabajo de síntesis ha demostrado ser una excelente herramienta de evaluación ya que evidencia con claridad suficiente, el nivel de conocimientos y destrezas adquiridas.

En la siguiente tabla pueden observarse los resultados de la evaluación entre los cursos 2005-06 y 2010-11.

\begin{tabular}{lrrr}
\hline & Intracampus & Intercampus & Total \\
\hline NP & 28 & 13 & 41 \\
\hline Susp. & 9 & 7 & 16 \\
\hline Aprob. & 32 & 25 & 57 \\
\hline Not. & 156 & 112 & 268 \\
\hline Sobresal. & 16 & 21 & 37 \\
\hline MH & 1 & 2 & 3 \\
\hline Alumnos & 242 & 180 & 422 \\
\hline
\end{tabular}

Tabla V. Calificaciones obtenidas entre los cursos 2005-06 y 2010-11 (elaboración propia a partir de datos facilitados por la UAB)

En general los resultados fueron satisfactorios y el número de alumnos que abandonaron la asignatura bastante reducido, lo que se consideró un éxito en lo que se refiere al seguimiento de la misma.

\subsection{Comunicación e interacción en el aula}

Algunos de los principales factores que pueden condicionar la comunicación en el aula virtual son la no presencialidad, la asincronía y la mediación del lenguaje escrito. Estos factores afectan positiva y negativamente. Por un lado, hacen que la comunicación, como ya se comentó, carezca de la inmediatez y la emotividad que caracterizan a la conversación presencial. Por otro, hacen que la comunicación se dé en un contexto de anonimato que potencia la participación. Tanto estudiantes como profesores cuentan, además, con mayor tiempo para reflexionar sus ideas y la forma de expresarlas, lo que resulta positivo porque contribuye no sólo al desarrollo de la capacidad de análisis sino también de la capacidad de síntesis (en el contexto virtual los mensajes deben ser claros, concisos y breves, sobre todo las contribuciones publicadas en los foros y otros espacios comunes de interacción).

Desde el punto de vista comunicativo, al docente se le plantean varios retos que deberá intentar superar contando, como instrumento, con la palabra escrita:

1. Combatir el distanciamiento que generan los espacios virtuales: Para ello se aconseja "humanizarlos" en la medida de lo posible. En este sentido, el uso de un lenguaje de tono cordial y próximo se considera lo más indicado.

2. Minimizar la incertidumbre suscitada por las asignaturas de tipo virtual en algunos alumnos: En "Surfear" esa incertidumbre suele detectarse al inicio del curso, principalmente a través de las contribuciones publicadas en el foro "Quién es quién".

3. Potenciar la participación y colaboración: Una comunidad de aprendizaje es operativa cuando existe un nivel de participación y colaboración adecuado para la creación y adquisición conjunta de conocimientos. Favorecer la participación es posible dejando constancia frecuente de la presencia del docente en el aula (publicación de noticias, sugerencia de actividades voluntarias o temas de debate, contribuciones en los foros, comentarios de apoyo que destaquen ideas de algún alumno, etc.). Cuando el alumnado percibe que el espacio virtual es algo cuidadosamente atendido, cuando intuye que su presencia o ausencia en el mismo no pasará desapercibida, sin duda, se hará más proclive a la participación.

4. Conseguir un óptimo cumplimiento de las normas de trabajo y convivencia establecidas: A través del lenguaje escrito no es siempre fácil lograr que el alumno comprenda y asuma las repercusiones que pueden tener determinadas actitudes y acciones en la buena marcha del grupo y en la labor del docente. En este sentido, la experiencia en "Surfear" ha evidenciado que los mensajes rigurosos o excesivamente exigentes por lo general tienen un efecto contraproducente y pueden incidir negativamente en la imagen que el grupo se haga del profesor, así como en el buen clima del aula. Cuando se dan situaciones o actitudes no deseadas, una expresión que argumente de forma sencilla el sentido y la lógica de las normas establecidas, así como los efectos que su incumplimiento provoca, suele bastar para lograr la 
comprensión de la mayoría de los estudiantes y evitar que se reiteren.

En general, todo docente con cierta experiencia conoce las estrategias comunicativas y lingüísticas que pueden hacer su discurso más efectivo. Se trata entonces de analizar las especificidades del entorno virtual y adaptarlas a ese contexto. Esto es algo que en ocasiones se logra a través de la propia intuición y el sentido común. No obstante, cabe señalar que el ejercicio profesional de este tipo de docencia constituye un insustituible laboratorio de aprendizaje y experimentación. La asistencia a cursos o actividades de formación específicas son también de gran ayuda.

\section{Conclusiones}

Las TIC han favorecido la creación de programas interuniversitarios virtuales de intercambio de asignaturas. Esto ha contribuido a la colaboración entre instituciones, al avance en el desarrollo de la universidad electrónica, así como a diversificar los conocimientos del alumnado.

La presencia de la formación en competencias informacionales es necesaria e ineludible en la educación superior. Estas competencias son la base del aprendizaje continuo a lo largo de toda la vida, que habrá de ayudar a los futuros profesionales a mantener en óptimo estado de actualización lo aprendido en las aulas universitarias.

Si bien es cierto que el carácter transversal de las competencias informacionales permite que la formación en las mismas pueda efectuarse desde un amplio abanico de materias, es conveniente que las titulaciones cuenten con asignaturas específicas. Asimismo que éstas sean impartidas por profesorado especializado.

La docencia virtual posee unas características propias y diferenciadoras de la presencial. Por ello, el profesorado debe afrontar el reto que ésta plantea contando con las competencias necesarias para impartirla de manera óptima. Sólo así será posible garantizar una enseñanza de calidad que contribuya a la excelencia de las instituciones universitarias.

Antes de cursar asignaturas virtuales, el alumnado debería haber sido instruido en el uso de la plataforma tecnológica y las herramientas utilizadas en su institución, asimismo en técnicas para el aprendizaje autónomo, comunicativas y de gestión del tiempo. Con ello se favorecería la correcta dinámica de las asignaturas, el propio proceso de aprendizaje y la adquisición de conocimientos.
5.1. Orientaciones para el ejercio de la docencia virtual

Para el ejercicio de la docencia virtual puede ser útil tener en cuenta las siguientes orientaciones que sintetizan las ideas tratadas a lo largo del presente trabajo:

- Planificación: Si ésta es necesaria en la enseñanza presencial, lo es más, si cabe, en la virtual. Hay que tener en cuenta que los estudiantes poseen distintas frecuencias de entrada en el aula, diferentes ritmos de estudio. Los cambios, las modificaciones en el calendario, la publicación de nuevas normas, etc., generarán desconcierto y tal vez lleve días el que sean conocidas o asumidas por la totalidad del grupo. Por ello conviene evitarlas en la medida de lo posible y a través de una adecuada planificación previa.

- Previsión: la docencia virtual depende de tecnologías que pueden plantear problemas imprevistos. Pensar en cuáles pueden ser los tales problemas, así como en posibles soluciones para el caso de que se produzcan, ahorrará complicaciones y posteriores pérdidas de tiempo durante el curso.

- Anticipación: el espacio virtual genera un distanciamiento que a veces provoca en el alumnado una incertidumbre superior a la que se da en contextos presenciales. En este sentido, es efectivo elaborar material explicativo suficiente y detallado sobre el funcionamiento de la asignatura y lo que es más importante, que dicho material se encuentre a disposición de los estudiantes desde el inicio. Se considera especialmente relevante dejar claras las normas de entrega de trabajos, comunicación, así como las relacionadas con la convivencia en el aula.

- Exigencia en el cumplimiento de la normativa que rige la asignatura: esto permite establecer en el aula el orden necesario para el óptimo desarrollo de los procesos de enseñanza y aprendizaje.

- Explotación de los recursos tecnológicos: es conveniente el uso intensivo de los recursos, prestaciones y utilidades que proporciona la tecnología para mejorar la calidad de la enseñanza y contribuir a la innovación docente.

- Multimedialidad: referida, en este caso, a la elaboración del material didáctico. Se aconseja aprovechar todo lo que ofrece la tecnología para crear documentos de trabajo que incluyan información de naturaleza diversa (textos, registros sonoros, audiovisuales, presentaciones de diapositivas, gráficos in-

Jiménez López, Angels. El ejercicio de la docencia virtual en la educación universitaria: consideraciones prácticas basadas en la experiencia de una asignatura para la formación de competencias informacionales. // 17:2 (jul.-dic. 2011) 49-62. ISSN 1135-3716. 
teractivos, etc.). Las nuevas generaciones están muy familiarizadas con el consumo de información a través de canales diversos y en un amplio abanico de formatos, por ello, conviene adaptar los materiales a esa realidad, favoreciendo así el aprendizaje.

- Normalización: en la medida de lo posible, conviene normalizar los procesos, procedimientos y la documentación elaborada para conferir claridad al espacio virtual y facilitar su comprensión por parte del alumnado.

- Asertividad: el docente es el máximo responsable de la asignatura y del aula. Como tal, su autoridad debe estar presente en el espacio virtual, aunque sólo contará para ejercerla en la mayoría de los casos, con la palabra escrita. Una comunicación asertiva, que sitúe su discurso en un punto justo de equilibrio se hace imprescindible para conseguir los fines propuestos y evitar reacciones adversas que afecten al buen ambiente del aula o a la percepción que el alumnado tenga de él.

\section{Notas}

(1) El estudio de Area, aun datando de 2001, proporciona datos cuantitativos y cualitativos que sirven de referencia y ayudan a conocer la historia del desarrollo de los campus virtuales en las universidades españolas.

\section{Referencias}

ACRL (Association of College \& Research Libraries), (2000). Normas sobre aptitudes para el acceso y uso de la información en la enseñanza superior. Chicago:
ACRL,2000. http://www.ala.org/ala/mgrps/divs/acrl/stan dards/informationliteracycompetencystandards.cfm ( 0110-2011).

Area, M. (dir.) (2001). La oferta de educación superior a través de Internet: Análisis de los campus virtuales de las universidades españolas. Informe. http://www.edulab .ull.es/campusvirtuales/informe/inicial.htm.(01-10- 2011).

Bautista, G. et al. (2006): Didáctica universitaria en entornos virtuales de enseñanza-aprendizaje. Madrid: Narcea, 2006. ISBN 84-277-1534-X.

Cabero, J. ; Romero, R. (2010). Análisis de buenas prácticas del e-learning en las universidades andaluzas. // Revista Electrónica Teoría de la Educación: Educación y Cultura en la Sociedad de la Información. 11:1 (2010) 283-309. http://tecnologiaedu.us.es/cuestionario/biblio vir/jca44.pdf. (09-11-2011).

Casamayor, G. (coord.) (2008). La formación on-line: una mirada integral sobre el e-learning, b-learning. Barcelona: Graó, 2008. ISBN: 987-84-7827-656-1.

Cepillo, M.A. (2010). Foros de debate virtuales. En: Sánchez González, M. P. (coord.). Técnicas docentes y sistemas de evaluación en educación superior. Madrid: Narcea, 2010, p. 49-52. ISBN: 987-84-277-1716-9.

CRUE-TIC (Conferencia de Rectores de Universidades Españolas); REBIUN (Red de Bibliotecas Universitarias) (2009). Competencias informáticas e informacionales en los estudios de grado. http://www.rebiun.org/doc/docu mento_competencias_informaticas.pdf (01-10-2011).

Matabay, R. (2008). El perfil del docente virtual. http://www.slideshare.net/rematabay/perfil-del-docentevirtual-presentation-801699 (09-10-2011).

REBIUN. Grupo de trabajo ALFIN. (2008). Guía de buenas prácticas para el desarrollo de las competencias informacionales en las universidades españolas. http://www. caul.edu.au/info-literacy/InfoLiteracyBestPractice.pdf. (02-10-2011).

Silva Quiroz, J. (2010). Enseñar en los espacios virtuales: de "profesor" a "tutor". // Revista electrónica de diálogos educativos. 19 (2010). http://www.umce.cl/ dialogos/ n19_2010/silva.swf.(30-10-2011). 